Mots. Les langages du politique

Dire l'altérité autochtone. Débats anthropologiques et indigénistes sur le sujet indien au Mexique (1940-1948)

Indigenous alterity into words. Anthropological and indigenous debates on the Indian subject in Mexico (1940-1948)

Describir la alteridad indígena. Debates antropológicos e indigenistas sobre los indígenas en México (1940-1948)

Paula López Caballero

CpenEdition

Journals

Édition électronique

URL : http://journals.openedition.org/mots/22987

DOI : $10.4000 /$ mots. 22987

ISSN : 1960-6001

Éditeur

ENS Éditions

Édition imprimée

Date de publication : 1 novembre 2017

Pagination : 145-164

ISSN : 0243-6450

Référence électronique

Paula López Caballero, « Dire l'altérité autochtone. Débats anthropologiques et indigénistes sur le sujet indien au Mexique (1940-1948) », Mots. Les langages du politique [En ligne], 115 | 2017, mis en ligne le 21 novembre 2019, consulté le 05 janvier 2021. URL : http://journals.openedition.org/mots/22987 ; DOI : https://doi.org/10.4000/mots.22987 


\section{Dire l'altérité autochtone. Débats anthropologiques et indigénistes sur le sujet indien au Mexique (1940-1948)*}

«Définir c'est, par essence logique, préciser les détails qui conviennent à un concept non seulement à un moment donné, mais pour toujours. Et la principale utilité de la définition repose précisément sur sa permanence.»

Alfonso Caso, 1948

En 1948 est inauguré, à Mexico, l’Institut national indigéniste, à la suite du

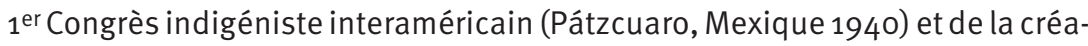
tion de l'Institut indigéniste interaméricain (1941), à Mexico. Dès lors, ces deux instituts, pris en charge par des anthropologues, marqueront le développement des politiques destinées aux populations identifiées comme indiennes au Mexique et aux Amériques ${ }^{1}$, liant ainsi intimement la discipline anthropologique aux politiques publiques. L'un des principaux problèmes auxquels se sont confrontés ces anthropologues-fonctionnaires était de déterminer qui était leur sujet d’intervention, d'établir qui étaient les «Indiens». Était-il donc possible qu'en pleine moitié du xxe siècle, près de trente ans après la Révolution et plus d'un siècle depuis l'Indépendance, les spécialistes chargés d' ' intégrer» ce qui était perçu comme diversité raciale ou culturelle en une seule nation ne puissent pas déterminer avec précision à qui ils devaient adresser leurs politiques publiques? En amont de savoir quelles politiques seraient les

* Ce texte a été élaboré dans le cadre du projet Pensar la alteridad en México, CONACYT-CB 242433. Je remercie les collègues du projet Rethinking Change, History and Indigeneity, du séminaire «Mobilités, gouvernance et ressources dans le bassin méso-américain » de l'unité de recherche Migrations et société (CNRS, UMR 8245; IRD, UMR 205; universités Paris-Diderot et Nice Sophia-Antipolis) ainsi que Benoît de l’Estoile et Álvaro Morcillo pour leurs utiles et généreuses suggestions.

1. Puisque l'objet même de cet article est la catégorie « indígena » (ou «Indio »), ces termes seront situés du côté de l'évidence empirique et non pas de celui des outils analytiques. Je restitue donc l'utilisation faite par les acteurs de l'époque de termes tels que «Indio» ou «Indígena» (en les traduisant par «Indien » ou « groupe indien »), sans adhérer aux contenus qu'ils y associent. 
plus efficaces pour atteindre l'unité nationale, le défi a été de savoir qui était indien et en quoi résidait l'altérité de ce groupe. Cette question, bien que pratiquement ignorée par l'histoire de l'anthropologie et celle des politiques indigénistes, s'est trouvée, en fait, au centre des débats de l'époque². Cet article rendra donc compte de cet intense débat, qui s'est tenu tout au long de la décennie de 1940 entre anthropologues et indigénistes 3 , mexicains et étrangers, principalement dans des articles publiés dans une importante revue de l'époque, América Indígena4.

Pour appréhender ces discussions, j'emprunte à l'histoire des sciences le concept d' "espaces de controverse » (Nudler, 2004)5, suggérant que les positions défendues ne sont pas antérieures au débat, mais que c'est justement dans le feu de l'échange que chaque posture s'affirme. Au croisement d'une histoire de la pensée anthropologique, d'une histoire institutionnelle de l'indigénisme et d'une histoire conceptuelle du sujet indien, la polémique reconstruite ici est historique et actuelle à la fois car, au cours de celle-ci, un certain nombre de traits toujours en vigueur de ce que signifie être indien prendront forme. La vitalité de ces idées jusqu'à nos jours permet de faire l'hypothèse que, malgré le rejet actuel des politiques indigénistes en faveur des rhétoriques multiculturelles, son empreinte est toujours présente dans les significations et valeurs attribuées au sujet indien et à son altérité.

\section{Indigénistes interaméricains vs anthropologues de Chicago}

La base agraire de la Constitution de 1917 issue de la Révolution (1910-1917) qui fonde l’État mexicain détermine en deux sens, au moins, les débats ana-

2. L'historiographie se concentre sur la période qui va de la création du ministère de l'Éducation publique en 1921 (dont le sigle est SEP en espagnol) à la fin du gouvernement Cárdenas en 1940 (cf. Knight, 1990, ou en français Favre, 1996). Ceux qui dépassent cette borne ne s’attardent pas sur la période ici étudiée : par exemple, Téllez Ortega, 1987, De la Peña, 2014. Mais voir Hewitt (1988) et Giraudo (2014) qui abordent des problèmes connexes. Voir aussi Giudicelli, 2010, Alvizuri, 2012, Canessa, 2012, 2014, ou Guyon, 2013 pour des questions similaires dans d'autres contextes.

3. Ce terme, largement utilisé à l'époque, faisait référence à tous ceux travaillant avec les populations rurales, la plupart d'entre eux étant des instituteurs ruraux, des pédagogues, des médecins, agronomes, etc.

4. Principale revue scientifique indigéniste et anthropologique de l'époque, publiée trimestriellement par l'Institut indigéniste interaméricain entre 1941 et 1999, avec une diffusion continentale et en espagnol. Sans dossiers thématiques, les varias sont composés de cinq à sept articles, d'une longueur maximale de cinq pages, sur deux colonnes. Elle comprend aussi un éditorial d'une à trois pages en anglais rédigé par son directeur, Manuel Gamio qui y soulignait, numéro après numéro, sa ligne éditoriale, bien que la revue ait publié des textes aux approches très différentes. Surtout pour les années ici étudiées la publication se faisait principalement par invitation et sans évaluation.

5. Nudler propose de comprendre l'histoire des sciences comme une succession de controverses plus que comme des périodes de vérités stables et cumulatives, comme semble le suggérer l'idée de paradigme (Kuhn). 
lysés ici. Premièrement, jusqu'aux années 1940, la question autochtone se réglait «au sein » de la question paysanne, privilégiant donc le facteur «classe» sur celui de l'« ethnicité». Par la suite, un virage conservateur se consolidera qui entamera, la décennie suivante, «l'institutionnalisation» de la Révolution. Une conséquence étant que, tant la classe comme critère d'action publique, que l'orientation marxiste de bon nombre d'anthropologues et indigénistes, deviendront alors implicites. Deuxièmement, la Constitution mexicaine de 1917 ne reconnaît pas de distinctions au sein de l'ensemble citoyen. De ce fait, l'Indien n'est pas un sujet de droits en tant que tel, mais plutôt en tant que paysan ('’article 27 établit le droit constitutionnel à l'accès à la terre). Le lien direct entre «identification » et «droit» n'est donc pas central dans ces débats, même si la constitution d'un champ d'intervention politique en est le substrat.

À partir de la lecture des presque trente numéros de la revue América Indígena, publiés entre 1941 et 1948 , trois types d'article se distinguent : des articles historiques (12\%), des articles ethnographiques (30\%) et ceux que j'appelle des «textes programmatiques» (58\%), c'est-à-dire, des essais plus «normatifs», qui discutent d'autres points de vue et dont les débats analysés ici font partie ${ }^{6}$. Les trois premiers articles, rédigés par le directeur du tout nouveau Institut indigéniste interaméricain, Manuel Gamio7, datent de 1942. Dans le premier, il pointe la nécessité, pour les institutions indigénistes, de déterminer «le nombre d'individus au sein de la population continentale qui peut être correctement classé sous le terme générique d'Indiens ». Car jusqu'à présent, les critères les plus courants sont la langue et la race, mais aucun d'eux n'est suffisant. Le premier, parce qu' «il laisse de côté [...] plusieurs millions d'individus qui parlent seulement espagnol mais sont Indiens en raison de leurs caractéristiques ethniques et culturelles». Quant au second, l'objection de l'auteur n'est pas conceptuelle - ce que signifie la race, si les races existent ou non, etc. - mais «technique» : déterminer la race demande «beaucoup de temps, de grands moyens financiers ». Il faut plutôt classer ces groupes « du point de vue de leur culture respective, autrement dit, selon les aspects que présente leur vie matérielle et intellectuelle» [1] ${ }^{8}$ (Gamio, 1942a, p. 17 et 18). Dans l'article suivant, il propose d'élaborer une «classification des caractéristiques culturelles différenciant les proprement indiennes, [...] les étrangères [...] et les mixtes» sur la base des objets se trouvant dans chaque foyer. Les premières

6. Base de données élaborée dans le cadre de ma recherche sur les politiques indigénistes des années 1940. D’autres débats publiés à América Indígena ont été analysés par L. Giraudo et J. Martín-Sánchez (2013).

7. M. Gamio (1883-1962) réalisa les premières fouilles archéologiques et les premiers travaux ethnographiques financés par l'État mexicain, entre 1917 et 1921. Il a également publié Forjando Patria (1916), qui deviendra l'un des piliers de la pensée indigéniste mexicaine. Son arrivée à l'Institut indigéniste interaméricain reste un sujet à étudier. Il le dirigera jusqu'à sa mort en 1962.

8. Toutes les traductions ont été faites par mes soins. Les chiffres entre crochets renvoient aux versions originales qui se trouvent en annexe de l'article. 
seraient reconnaissables par leur origine précolombienne ou par leur « développement biologique déficient [correspondant] à des étapes d'évolution anachroniques et inférieures » [2] (Gamio, 1942b, p. 15). On pourrait alors déterminer « objectivement » à quel type de culture appartiennent ses membres. Dans le troisième article, M. Gamio s’interroge : «Qui détermine combien et [quelles caractéristiques] doivent être conservées, écartées, corrigées ou substituées, et combien et lesquelles doivent être introduites? Convient-il de laisser une tâche aussi difficile au seul critère de l'Indien lui-même [...] ? ». Sa réponse est non. Dans certains domaines, comme la santé ou l'éducation, le «critère conventionnel des Indiens » doit se soumettre au «critère scientifique » de l'anthropologue [3] (Gamio, 1942c, p.17 et 22).

Cet ensemble d'articles ont servi de base à une sorte de norme de l'histoire de l'anthropologie mexicaine selon laquelle la culture aurait supplanté la race au sein de cette discipline. Certes, M. Gamio accorde une place privilégiée à la culture matérielle. L'imbrication entre les différents termes qu'il emploie attire cependant l'attention : développement biologique, héritage, caractéristiques culturelles et pauvreté. Un amalgame entre ces notions, où M. Gamio «biologise», pour ainsi dire, la culture et «culturise » la pauvreté9.

On peut supposer que ces articles constituent une réponse à un texte publié par Robert Redfield ${ }^{10}$ en anglais en mars 1940 et dont quelqu'un comme M. Gamio, en contact avec les universitaires nord-américains, peut avoir eu connaissance. Dans un contexte intellectuel, où la réalité sociale du monde rural mexicain était conçue en termes de "groupes", homogènes en leur sein et clairement différenciables entre eux, l'article de R. Redfield détonne, par contraste. Le texte s'ouvre sur une phrase sans ambages :

Dans un pays tel que le Mexique, où l'intermixité raciale a largement eu lieu, il est possible d'estimer les montants relatifs de sang indien et de sang blanc, mais non pas [...] de dénombrer les Indiens comme un groupe socialement reconnu, tel que l'on fait avec les Noirs [Negroes] aux États-Unis, car dans la plupart des régions [...] les Indiens ne sont pas ainsi définis [...] personne n'est de façon définitive un Indien. [4] (Redfield, 1940, p. 132)

Ensuite, à la différence de M. Gamio, R. Redfield estime que le problème avec la race n'est pas seulement technique ; pour lui, c'est une donnée qui n'apporte aucune information positive dans une société comme celle du Mexique. R. Redfield souligne en outre que le problème principal ne réside pas dans la détermination de l'origine des éléments de la vie sociale et culturelle de

9. L’idée de métissage (un mot issu du champ biologique par ailleurs), centrale à l'idéologie nationaliste postrévolutionnaire, n'échappe pas à cette pensée racialiste car elle pose le métis comme le résultat du mélange entre deux races.

10. Robert Redfield (1897-1958) a étudié et travaillé à l'Université de Chicago. En plus d'être une autorité dans la discipline, il sera l'intermédiaire incontournable de tout projet nord-américain au Mexique, notamment à travers les fondations (Rockefeller, Carnegie). 
chaque groupe, mais dans la reconnaissance de la fragmentation sociale qui règne dans le pays. Ainsi, plus qu'une question de culture ou d'évolution, «le "problème indien" au Mexique consiste à transformer beaucoup de sociétés villageoises [folk societies] en une seule nation ». [5] (Redfield, 1940, p. 138)

Les deux anthropologues s'accordent sur le fait qu'en raison des conditions de vie des Indiens, la nation souffre de fragmentation. Leur divergence, bien que subtile, est que pour M. Gamio le principal frein à leur développement réside dans leur culture (qui, paradoxalement, reste aussi leur principale vertu). Le changement doit donc s'opérer en modifiant partiellement celle-ci tout en conservant des aspects appréciés des cultures indiennes. R. Redfield, quant à lui, estime que le frein au développement réside dans les capacités limitées de l'État pour accéder à des régions isolées. La transformation doit s'opérer sur les conditions économiques et sociales de la population, même si cela peut impliquer une évolution de nature culturelle.

Mais le champ de la pensée indigéniste ne se limite pas à ces deux positions. L'article de R. Redfield sera traduit et publié dans la Revista Mexicana de Sociología en 1942 (et non pas, ce qui est significatif, dans América Indígena). Le directeur de cette publication, Lucio Mendieta y Núñez ${ }^{11}$ réagira également à ces arguments. À l'opposé de Redfield, il soutient que de nombreux groupes indiens « sont restés dans un isolement tel qu'il leur a été possible de perdurer comme groupes ethniques différenciés. D’autres races indiennes se sont mélangées entre elles, mais le résultat [...] est toujours un type indien » [6] (Mendieta, 1942, p. 65 et 66) ${ }^{12}$. Et de conclure : «Identifier au Mexique race indienne et classe sociale n'amène qu'à des tergiversations intéressées [...] car cela évince, artificiellement, l'un des termes principaux : celui de la race qui y joue un rôle prépondérant» [7] (Ibid., p. 67).

Assurément, cette opinion représente le pôle extrême du débat, en considérant comme innée l'altérité de ceux qui sont identifiés comme autochtones. Toujours est-il que cette conception biologisante de la race ne suscite pas de réactions ni de critiques dans les publications. Bien que quelqu'un comme M. Gamio ne défende pas cette idée de race comme principal «marqueur d'altérité», il ne nie pas non plus son existence comme donnée positive ni comme facteur d'explication.

Un quatrième anthropologue intervient dans América Indígena. Il s'agit du texte «Ethnic relations in Guatemala» du jeune anthropologue Sol Tax¹3. II

11. L. Mendieta (1895-1988) étudie à l’Escuela Nacional de Jurisprudencia en 1915 et, dès 1917, travaille aux côtés de M. Gamio à la Direction d'anthropologie. En 1939, il crée et dirige l'Institut de recherches sociales de l'UNAM (1939-1965), la Revista Mexicana de Sociología la même année et les Cuadernos de Sociología (1947).

12. Il apparaît que pour L. Mendieta, «groupe ethnique» et « race» étaient des termes interchangeables.

13. S. Tax (1907-1995) avait obtenu son doctorat en 1934 et sera un proche collaborateur de R. Redfield à l'Université de Chicago. Il séjournera au Mexique entre 1942 et 1943 comme professeur 
n'est pas impossible que cette étude soit la première, du moins dans le champ latino-américaniste, à parler de «relations » ethniques ${ }^{14}$. Le type de publication qui prédomine à l'époque suggère que jusqu'alors il n'y avait d' «ethnique» (le plus souvent compris comme "race») que les groupes et leurs caractéristiques. La nouveauté introduite dans le titre synthétise, en effet, une approche rafraîchissante du problème indien tel qu'on l'entendait alors.

S. Tax conforte la position de son mentor, R. Redfield, et débute son argumentaire en affirmant que «pratiquement partout au Guatemala, les distinctions contemporaines ne se fondent pas sur la race biologique » [8] (Tax, 1942, p. 44). Le « problème indien » n'est donc pas une question « raciale » comme on pourrait l'entendre aux États-Unis, puisque les éléments qui permettent de distinguer un Indien d'un non-Indien sont «indépendants de l'héritage physique ou biologique ». D’où le fait que le problème indien au Guatemala soit, principalement, une question d'inégalité et de manque d'opportunités :

Il y a bien un problème indien au Guatemala, mais pour le comprendre et le résoudre, il faut d'abord nettoyer de notre esprit l'idée qu'il s'agit d'un problème de race. Il est vrai que partout ceux qui sont appelés «Indiens» au Guatemala, sont des descendants sanguins [blood descendants] des aborigènes américains : il est vrai aussi que la plupart des non-Indiens au Guatemala ont plus ou moins du sang européen. Il est faux que ces facteurs soient socialement importants. [9] (Ibid.; nous soulignons)

S. Tax ne nie pas que «la race » existe, ni qu'au Guatemala ceux qui sont identifiés comme Indiens soient méprisés sur une base phénotypique ou de couleur de peau. Son argument, cependant, est que l'altérité se définit sur la base de critères sociaux plus que biologiques : la langue est donnée comme un indicateur fondamental, tout comme le type de vêtements et la participation à la vie politico-religieuse des villages. L'auteur reconnaît le caractère vague de ces indicateurs mais, pour lui, le point important est que « tous les critères mentionnés sont indépendants de l'héritage physique [...] toute la question du sang [...] est insignifiante dans la distinction faite dans la pratique ». Et de conclure : «Une fois compris [cela] il ne semblera plus étrange que l'un des deux frères puisse être un Indien et l'autre, peut-être même vivant dans le même village, un Ladino ». D'où que «le passage d'un groupe à l'autre est possible; plus encore, il tend à devenir automatique. Un Indien qui perd ses caractéristiques indiennes est un Ladino» [10] (Tax, 1942, p. 45 et 46) 15. Pour terminer,

de l'École nationale d'anthropologie. Ses recherches portent sur l'organisation sociale et les relations économiques au sein de différents groupes linguistiques mayas, notamment au Guatemala.

14. Il y a peut-être ici un lien avec le projet sociologique de Talcott Parsons qui dès 1940 conçoit un «Département des relations sociales» à Harvard.

15. Il serait inexact d'interpréter cet argument comme la défense d'une certaine «démocratie raciale » latino-américaine, représentation usuelle lorsque l'on compare l'idée de race auxÉtatsUnis et en Amérique latine. Pour une critique de cette opposition, voir Wade, 1993. 
il soutient que l'origine du «problème indien » ne réside pas dans un élément intrinsèque aux groupes ainsi désignés (y compris « leur » culture), mais dans le contexte national d'inégalité. Ainsi, le plus urgent en termes de transformation sociale serait de défaire l'association entre pauvreté, exclusion et indianité.

Si l'on tient compte de la chronologie, aujourd'hui consensuelle, selon laquelle la race n'a pas été un critère central des politiques indigénistes et, surtout, que dans les années 1940 elle avait déjà été abandonnée, les réactions à l'encontre des arguments de R. Redfield et de S. Tax se révèlent surprenantes. Les éditeurs d'América Indígena ont décidé d'inclure, à la fin de cet article, une lettre-commentaire rédigée par John Collier (1884-1968), directeur de l'Indian Affairs Bureau (États-Unis) et principal acteur politique de l'indigénisme interaméricain, dans laquelle il rejette d'un revers de manche les hypothèses de l'anthropologue. Pour ce fonctionnaire, «l'article cherche à abolir le facteur d'indianité, en parlant d'un pays où la population est largement indienne [...] D’ailleurs, c'est ainsi que le problème indien a été longtemps évincé » [11] (Collier dans Tax, 1942, p. 47). En d'autres termes, pour lui, la reconnaissance de la spécificité des problèmes vécus par les Indiens est un progrès et la proposition de S. Tax serait une régression. Il établit en outre un parallèle plus ou moins linéaire entre « race » et « indianhood » dans la mesure où pour lui, nier, comme le fait S. Tax, qu'il existe un problème racial, revient à nier qu'il existe un problème indien. Plus encore, l'interprétation que donne J. Collier semble inverser tout l'argumentaire de S. Tax :

Dire avec insistance que l'Indien n'existe pas ou qu'en tant qu'Indien il doit être ignoré à moins que son identification biologique, linguistique, sociale, légale et économique s'emboîtent exactement, suppose une incompréhension désespérante du sujet. [12] (Collier, dans Tax, 1942, p. 47)

Enfin, il accuse S. Tax de contredire aussi bien les thèses soutenues par l'Institut indigéniste interaméricain, que les contributions présentées à Pátzcuaro, et «toute l'expérience de pays tels que le Mexique ou les États-Unis, et audelà... » [13] (Ibid., p. 48). Implicitement, J. Collier confirme donc que la critique de S. Tax donnait dans le mille : son analyse de la situation ethnique au Guatemala déstabilisait et remettait en question tout le projet d'un indigénisme interaméricain unifié, si cher à J. Collier (et à M. Gamio) ${ }^{16}$.

Tant et si bien que dans le numéro suivant d'América Indígena, les éditeurs publient l'avis de plusieurs collègues sous le titre «Comments on Dr. Tax's Article». Le premier à intervenir est R. Redfield, qui, dans une missive soignée, propose trois arguments principaux pour soutenir l'exposé de S. Tax:1) La situation des Indiens au Guatemala n'est pas la même qu'aux États-Unis, notamment

16. Le premier projet d'intervention de l'Institut indigéniste interaméricain a eu lieu, précisément, au Guatemala à cette époque (Projet de la zone onchocercose au Guatemala). Peut-être la virulente réaction des deux indigénistes contre $S$. Tax répondait-elle aussi à ce contexte politique. 
car il y est plus difficile d'identifier un «problème indien » indépendant des problèmes sociaux en général ; 2) Aux États-Unis, les Indiens sont relativement peu nombreux et vivent dans une forte ségrégation; un programme spécial semble alors plus clairement justifié ; par contre, au Guatemala, la population défavorisée est majoritairement indienne; un programme de développement social général bénéficierait donc nécessairement à ces groupes; 3) Au Guatemala, les habitants ruraux - Indiens et Ladinos - peuvent être perçus comme égaux par les habitants de la ville. Selon R. Redfield, c'est impossible aux États-Unis car

les distinctions entre groupes qui importent au Guatemala sont des différences de culture. Elles ne sont pas des différences de race [...] Aux États-Unis il y a une barrière sociale entre groupes raciaux qui se fonde sur la conscience de la différence de sang entre les membres d'une race et ceux d'une autre. Cette barrière est très forte dans les relations Noirs-Blancs; elle est présente dans les relations IndiensBlancs. Elle est pratiquement absente dans la majeure partie du Guatemala. [14] (Redfield, Collier, Sady et Presnall, 1943, p. 84 et 85 ; nous soulignons)

Dans les termes de mon propre cadre d'analyse, R. Redfield veut montrer que les «marqueurs d'altérité», ces données sociologiques qui permettent d'identifier quelqu'un comme «autre » (en l'espèce, comme indien), et leurs effets ou conséquences, sont contextuels, variant d'un pays à l'autre. Il était alors capable de se distancer de son propre «régime d'altérité » (états-unien) et de comprendre que J. Collier était en train de le transposer sans aucun recul au contexte guatémaltèque ${ }^{17}$. Il s'agissait donc de savoir dans quelle mesure le régime d'altérité qui fonctionnait aux États-Unis et semblait s'imposer au sein de l'Institut indigéniste interaméricain pouvait s'appliquer à l'Amérique latine.

Suit une très brève réponse de J. Collier (deux paragraphes), dans laquelle il insiste sur le fait que l'inégalité entre Indiens et Ladinos dépasse le seul aspect socioéconomique, car la culture indienne n'est pas valorisée. Le bienêtre général dont parle $S$. Tax est donc nécessairement posé en termes de valeurs ladinas (non indiennes). Mais sa critique centrale repose sur le fait que S. Tax «semble présumer qu'il n'y a aucune valeur interne ou organique au sein de la culture ou de la vie indienne » [15] (Redfield, Collier, Sady et Presnall, 1943, p. 86), d'où l'on peut déduire que pour J. Collier ces valeurs propres aux cultures indiennes sont une réalité à partir de laquelle l'intervention indigéniste doit être conçue et mise en œuvre.

S'exprime aussi un personnage peu connu de nos jours mais qui occupait une place relativement importante dans le milieu indigéniste des années 1940 : Emil Sady ${ }^{18}$. C'est lui qui classe les positions du débat en deux groupes de pro-

17. Sur le terme « régime d'altérité », voir López Caballero 2012 et 2016.

18. Il était le représentant, au Mexique, de l'Institut indigéniste des États-Unis, créé en 1941, mais qui a toujours été subordonné à l'Indian Affairs Bureau dirigé par J. Collier. E. Sady était en fait son subordonné et allié au Mexique. 
fessionnels : les anthropologues - S. Tax, R. Redfield - et les administrateurs - J. Collier et M. Gamio, bien que ce dernier ne soit pas nommé (Ibid., p. 88). Chacun des groupes ayant leur conception respective du changement : pour les premiers, toute innovation implique de changer les éléments qui tendent à maintenir une société intégrée; pour les seconds, « en faveur de la diversité culturelle ", il est possible d'introduire de nouveaux éléments sans déstructurer la culture. Au fond, me semble-t-il, ce qui les distingue est la valeur que chacun accorde à cette "culture » : pour les «administrateurs", si cette dernière se «perd», tout est perdu et justice ne sera pas faite puisque les groupes disparaîtront. Pour les «anthropologues », le changement n'est ni positif ni négatif, simplement inévitable, si l'on répond aux besoins les plus élémentaires, au lieu de donner la priorité à la « conservation» de la culture.

À la fin de cette année, Julian Steward ${ }^{19}$ publie un article dans lequel il tâche de proposer une position intermédiaire. C'est une erreur, dit-il, de considérer que lorsque les «administrateurs » souhaitent protéger « les valeurs distinctives indiennes », ils se réfèrent aux éléments de la culture précolombienne. Une fois éclaircie cette confusion, l'opposition entre les deux groupes n'en est plus une:

Si l'indianité dont parlent les [administrateurs] signifie [...] quelque chose qui se développe au sein de traditions natives et ne se maintient pas de façon statique ou même stable, je ne vois pas de conflit avec les anthropologues. Puisque ces derniers n'affirment pas que les forces acculturationnistes [acculturative] vont réduire l'Indien au point mort de l'homogénéité nationale. [16] (Steward, 1943, p. 324)

Pour synthétiser l'état de la controverse à ce moment-là, elle concerne essentiellement la façon de comprendre l'altérité indienne (comme une propriété, un degré de développement biologique, une race, un système culturel ou une position sociale) et sa transformation (inévitable, unidirectionnelle, orientable). Au cours de la polémique, entre l'altérité comme race telle que L. Mendieta la pose et la culture biologisée dont parle M. Gamio, par exemple, il n’y a pas de différence de type mais de degré. Les idées de L. Mendieta pouvaient donc être entendues et validées dans l'espace de cette controverse, même si elles restent en retrait. En ce qui concerne les idées défendues par R. Redfield et S. Tax qui, dans une certaine mesure, perçoivent l'altérité des Indiens comme une donnée relative, elles fonctionnent comme des déclencheurs pour affirmer, avec plus d'insistance, l'importance que le critère « culturel» (mais quand même inné) comme marqueur d'altérité avait pour les fonctionnaires de l’indigénisme interaméricain. La distinction entre scientifiques et administrateurs en est également un résultat.

19. Anthropologue nord-américain (1902-1972), formé par Alfred Kroeber et Robert Lowie à l'Université de Californie. Entre 1935 et 1946, il édite le Handbook of South American Indians et travaille pour la Smithsonian Institution. Entre 1946 et 1953, il enseigne à l’Université de Columbia, avant de diriger le département d'anthropologie de l'Université d'Illinois à Urbana-Champaign. 


\section{Entre la race et la culture, la «communauté indienne»}

Le débat reprend en avril 1945, avec un article publié par l'anthropologue Oscar Lewis ${ }^{20}$ et Ernest Maes, représentant de l'indigénisme nord-américain en Amérique latine. L'Institut, reconnaissent ces auteurs, doit commencer par préciser comment il définira l'Indien. Ils proposent alors « une définition qui sera pratique [pour recueillir l']information statistique et cadastrale, puis fonctionnelle vis-à-vis des objectifs et raison d'être de l'Institut indigéniste interaméricain ». Celle-ci doit rendre compte, non pas des propriétés singulières des Indiens ou, pour reprendre les termes des auteurs, des «caractéristiques déterminantes de ce qu'est l'autochtone [autochtonous]» mais des «déficiences économiques et sociales les plus aigues dont souffrent ces groupes sociaux» [17] (Lewis et Maes, 1945, p.108, 113 et 117). Cette proposition, qui pourrait être comprise comme le simple passage de l'idée de "culture » à celle de "classe », est intéressante car elle ne renonce pas, comme le fera le marxisme, ou la sociologie classique, à la catégorie «Indien ». En effet, il s'agit plutôt d'une mutation à l'intérieur même de la catégorie. Pour cela, les auteurs opèrent une sorte de jeu d'équilibriste pour tâcher d'articuler une notion d'Indien fondée sur l'altérité - centrale pour l'Institut indigéniste interaméricain - avec une notion pratique en termes d'administration publique :

Suivant notre critère, lorsqu'un groupe a le maximum de besoins et déficiences quantitatifs [...] en même temps que le maximum de déficiences qualitatives, nous sommes en présence d'un groupe qui pourra être appelé indien. [18] (Ibid., p. 115)

Ils finissent toutefois par accepter, implicitement, que la position socioéconomique n'est pas suffisante pour caractériser les populations autochtones, mais que c'est l'histoire d'oppression coloniale qui les détermine.

En conclusion, malgré ce retour à l'idée d'une constante fixe qui serait le propre des Indiens - l'oppression coloniale - le déplacement qu'ils proposent n'en est pas pour le moins innovant au sein du débat : il ne s'agit plus désormais de savoir où se situent les limites qui séparent l'Indien du non-Indien, ni en quoi réside l'altérité des personnes identifiées comme indiennes, mais quelles données peuvent être utiles pour mener une action pratique dans le cadre de l'indigénisme interaméricain. Glissement qui irait d'une définition "attributive» (de ce qui serait «propre» à l'Indien) vers une définition "d'usage », élaborée principalement en fonction de ceux qui l'utiliseront.

20. O. Lewis (1914-1970) obtient son doctorat à l'université de Columbia en 1940 après quoi il vit au Mexique et travaille à l'Institut indigéniste interaméricain. Ses deux principaux ouvrages sont Life in a Mexican Village: Tepoztlán restudied (1951) et Five Families: Mexican Case Studies in the Culture of Poverty (1959). 
Deux autres articles sont publiés en janvier et juillet 1947 par le jeune anthropologue autodidacte Julio de la Fuente ${ }^{21}$. Ils peuvent être considérés comme un premier effort de synthèse et seront traités ici de manière conjointe. J. de la Fuente ne fait pas explicitement allusion au débat qui, à cette époque, durait déjà depuis cinq ans. Cependant, les éléments et constructions argumentatives qu'il propose y renvoient avec suffisamment de certitude pour les inclure dans l'analyse.

Pour commencer, l'auteur prend ses distances avec l'idée selon laquelle «il est nécessaire [...] de définir avec précision, premièrement, les groupes qui doivent être considérés comme indiens », comme M. Gamio le proposait, et se rallie à la proposition d'O. Lewis et $\mathrm{E}$. Maes de partir des besoins socioéconomiques de la population pour établir à qui doit s'adresser la politique indigéniste. La raison qu'il donne est en effet novatrice : «Il n'est pas toujours opportun de s'adresser à l'Indien comme tel [car beaucoup d'entre eux] sont devenus des non-Indiens » [19] (Fuente, 1947a, p.63 et 64). Il convient donc d'adopter un critère qui tienne compte de cette évolution. Le reste de l'article constitue une argumentation en faveur de cette thèse.

J. de la Fuente reprend la critique faite par R. Redfield à l'encontre du critère racial en signalant que celui-ci a une signification très limitée en raison de la longue histoire de métissage de la population mexicaine. Cependant, dans une possible concession faite à M. Gamio, J. de la Fuente accepte que les «attitudes raciales» (ou racistes dirions-nous sans doute aujourd'hui), existent effectivement envers certains traits phénotypiques ou certaines coutumes. Il critique ensuite le critère culturel en raison de son caractère statique. Ce critère empêche, surtout, de rendre compte de ce qu'il appelle le «pase del Indio » pour signifier un processus où « "l'Indien” ne se considère pas lui-même comme tel ou bien a appris à se considérer comme un paysan, un travailleur ou un natif d'une région spécifique. „22 Enfin, tout comme S. Tax l'esquissait, pour J. de la Fuente la manière dont les individus catégorisés comme Indiens se «sentent» indiens, constitue un critère central de l' "indianité». Il conclut en distinguant indianité et culture, pour associer la première à l'appartenance régionale : «Ce qui tendrait à demeurer serait l'indianité dérivée d'un lieu ou d'une région »[20] (Fuente, 1947a, p.68 et 69).

Dans le deuxième article sa position semble, toutefois, se nuancer. Si le processus selon lequel un Indien cesse de l'être existe certes au Mexique, il

21. J. de la Fuente (ca.1905-1966) participe dès les années 1920 aux politiques postrévolutionnaires. En 1932 il obtient un poste d'instituteur rural et il mène en autodidacte des recherches sur l'organisation sociale de Yalalag (Oaxaca). Il se forme à l'anthropologie dans les années 1940, comme assistant de l'anthropologue britannique Bronislaw Malinowski, puis à l'École nationale d'anthropologie et en suivant divers enseignements aux États-Unis. Il entre dans l'administration indigéniste au cours de la décennie suivante.

22. Pour l'auteur, les Zapotèques de l'isthme du Mexique qui préfèrent être nommés selon la région dont ils proviennent - Juchitecos, Tehuanos - constituent un exemple de ce glissement. 
ne faut pas pour autant en conclure qu'il s'agisse d'un phénomène généralisé et homogène dans tout le pays, ni que cela se produise avec une telle rapidité que l'on puisse parler purement et simplement de la « disparition de l'Indien ». Bien que certains domaines de la culture indienne évoluent, l'anthropologue reconnaît qu'il existe des caractères qui «font [des Indiens] des sujets particuliers, distincts de la population "paysanne" et plus encore de la population urbaine » [21] (Fuente, 1947b, p. 212). D’où l'idée que des institutions telles que l'Institut indigéniste interaméricain soient indispensables pour mettre en place des politiques spécialement conçues pour ces populations.

L'altérité comme une constante fixe réapparaît ici aussi - en accord avec les idées de Gamio sur l'Indien - même si cette fois-ci ses contenus ne sont pas explicités. Il est étonnant, cependant, que ces affirmations servent à l'auteur pour soutenir l'argumentaire d'O. Lewis et $E$. Maes, et même pour contredire tacitement le directeur de l'Institut indigéniste interaméricain. Malgré la persistance de caractères «indiens », dit J. de la Fuente, le nombre d'éléments culturels qui sont en train de changer indique que le critère qui doit régir la politique indigéniste est celui des déficiences et des besoins de la population. Une politique indigéniste efficace doit donc s'adresser tant à ceux qui sont explicitement identifiés comme «Indiens» qu'à ceux qui ne le sont pas (Fuente, 1947b, p. 215).

En somme, les apports de J. de la Fuente sont pluriels : la centralité donnée au processus de changement massif que ces sociétés connaissaient, au détriment du critère culturel; le déplacement qui va de l'altérité-comme-culture à l'altérité-comme-appartenance régionale; l'importance de l'auto-perception. Compte tenu de la proximité entre ses critiques et celles des anthropologues nord-américains, pourquoi les arguments de J. de la Fuente n'ont-ils pas connu le même sort que ceux de Tax et Redfield? Quel déplacement s'est-il opéré dans l'argument de J. de la Fuente qui le rendait audible, valide?

Cela est peut-être dû à la reconnaissance de l'existence des Indiens comme groupes clairement identifiables, ainsi que la nécessité de mettre en place des politiques spécifiques à ces derniers, malgré les critiques qu'il reprend à l'encontre de ce genre de distinction ${ }^{23}$. En fait, ses deux articles sont significatifs précisément parce que l'auteur réussit à y concilier ce qui jusqu'alors paraissait incompatible: d'un côté, l'acceptation de l'existence d'un sujet indien avec des caractéristiques propres et, de l'autre, l’idée que pour être efficace, l'indigénisme doit dépasser cette définition culturelle ou attributive du sujet indien, en faveur d'une définition pratique qui s'adresse à tous ceux qui en ont besoin.

Si J. de la Fuente est celui qui «traduit» les idées des anthropologues de Chicago en les déplaçant juste ce qu'il faut pour pouvoir continuer à les affirmer

23. Sans compter, bien entendu, que J. de la Fuente ne disposait pas en 1947 du même statut ni du même poids académique et symbolique que S. Tax, ni, cela va sans dire, que R. Redfield. 
et en même temps les rendre audibles au milieu indigéniste, M. Gamio, quant à lui, intervient la même année, peut-être en réaction à celui-ci. Le contexte d'après-guerre s'immisçant toujours plus dans la revue, il dénonce ceux pour qui la population rurale doit recevoir un traitement égal, indépendamment de son indianité. Et de s’interroger :

Est-il logique et possible que du seul fait que les deux groupes d'individus [Indiens et non-Indiens] se débattent dans des conditions de misère égales ou analogues, il soit plus efficace de leur appliquer la même méthode et le même traitement? [...] Il est évident que cela se révèle impossible, qu'il faille formuler des traitements différenciés pour ces deux groupes et que le problème de l'Indien existe et a toujours existé [...]. [22] (Editorial, 1947, p. 283)

Ces considérations reprennent presque mot pour mot ce qui était affirmé au début de la décennie en réactivant l'idée d'une altérité qui serait intrinsèque aux populations indiennes. Le changement, certes subtil, réside effectivement dans le fait que le terme « race » s'estompe de plus en plus et que l’idée de «besoins» occupe désormais une place qu'elle n'avait pas auparavant.

L'actualisation de ces idées sera, cependant, l'œuvre, non pas de M. Gamio, mais d'un autre contemporain, lui aussi scientifique et fonctionnaire public : A. $\mathrm{Caso}^{24}$. Ce dernier fait avec les idées de M. Gamio l'équivalent du travail de J. de la Fuente avec celles de S. Tax et R. Redfield. Son article «Définition de l'Indien et de l'Indianité », pièce centrale du canon anthropologique et indigéniste mexicain, publié pour la première fois en octobre 1948, doit ainsi être compris comme héritier de ce débat 25.

A. Caso propose tout d'abord une réflexion presque philosophique sur la difficulté de définir un processus, une transformation, à la différence d'une idée stable ou d'un objet fixe (voir l'épigraphe de cet article). Reprenant ainsi l'un des points centraux de J. de la Fuente (mais sans le citer), il se confronte au défi de définir - et donc fixer - un phénomène changeant. Il reprend la critique du critère racial ou du « sens biologique », en grande mesure en raison du «mélange de l'Indien avec l'Européen » et l'illustre avec un exemple similaire à celui employé par S. Tax : «Dans un même village, dans une même famille, l'un des enfants peut présenter des caractéristiques somatiques indiennes qui font défaut à l'autre [enfant] » [23] (Caso, 1948, p. 87). Avec la différence fondamentale que S. Tax parlait de traits sociologiques (vêtement, langue, etc.) et A. Caso du phénotype!

24. A. Caso (1896-1970), archéologue, a découvert le plus riche tombeau précolombien à Monte Alban en 1929. Il est fondateur et premier directeur de l'Institut national d'anthropologie et d'histoire chargé de la protection du patrimoine national (1939) et de l'École nationale d'anthropologie (1942). Il a été recteur de l'Université Nationale (1944-1945) et enfin le fondateur de l’Institut national indigéniste (1948), qu'il dirigera jusqu'à sa mort en 1970.

25. Il s'agit d'un des textes les plus cités en la matière. Il paraît au moins dans un recueil de textes indigénistes datant de 1958, ainsi que dans un autre de 1971 et encore un autre de 1978. En plus de celles des indigénistes de l'époque, Google Scholars dénombre plus de 300 mentions de ce texte. 
A. Caso reconnaît le critère linguistique comme une donnée centrale pour déterminer si une personne est indienne. Mais il y ajoute l'élément que J. de la Fuente avait signalé : «La conscience d'appartenir ou non à un groupe indien est [...] le marqueur le plus important [...] mais [...] le plus difficile à capter » [24] (Ibid., p. 89). Ce quatrième critère, qu'il appelle «psychologique », constitue la base de sa définition de l'Indien, malgré - ou grâce à - la circularité de sa définition :

Est Indien celui qui se sent appartenir à une communauté indienne [...] Par conséquent [...] ce qui importe de déterminer dans le cadre d'une politique indigéniste [...] est, fondamentalement, la communauté indienne [comprise comme] celle où prédominent des éléments somatiques non-européens [...] et qui a un sens social de communauté isolée». [25] (Ibid., p. 90 et 91; nous soulignons)

Le nouveau sujet d'intervention de l'indigénisme défini, reste à déterminer ses besoins : «les grands problèmes de l'Indien, du moins au Mexique, ne sont pas seulement économiques, mais fondamentalement culturels », d'où sa conclusion : «ce que nous devons apporter à l'Indien [...] c'est la culture » [26] (Caso, 1948, p. 92).

Pour synthétiser, les préoccupations centrales de M. Gamio s'actualiseront dans la version d'A. Caso qui alimente ces arguments grâce aux critiques et aux apports de J. de la Fuente, S. Tax et R. Redfield. Ce texte peut alors être compris comme la «traduction » des idées des «anthropologues», au langage et au vocabulaire des «indigénistes » : le changement culturel et l'auto-identification proposées par les premiers servent désormais à défendre la nécessité, proclamée par les seconds, d'une définition de l'Indien et la place centrale de la culture - comprise comme un ensemble d'éléments qui doivent être modifiés ou préservés - dans cette définition. Au centre de cet emboîtement, la communauté devient le nouveau critère principal pour déterminer l'altérité de ces populations et pour la transformer.

\section{Instituer l’altérité indienne}

S’intéresser aux catégories et à leur historicité permet de les constituer en unités analytiques per se, et de s'interroger sur les différents contenus ayant été associés à un même vocable, au lieu de reproduire des définitions anhistoriques. Il est ainsi possible de focaliser l'analyse dans les usages que les acteurs font des catégories, leurs tensions, leurs mutations ${ }^{26}$. Cette approche permet de montrer comment chaque position se renforce «au feu » des débats euxmêmes. Au cours de ces discussions, chaque approche subit des modifications,

26. Sur les catégories comme objet historique, voir entre autres Bernard Lepetit (2013), et le numéro de la revue Raisons pratiques dédié à ce problème (Fradin, Quéré et Widmer, 1994). 
que ce soit en se radicalisant ou, la plupart du temps, en rejetant ou en reformulant les éléments les plus facilement critiquables de chaque point de vue.

La polémique que j'ai analysée montre comment les années qui vont du Congrès de Patzcuaro à la création de l'Institut national indigéniste sont fondamentales dans le processus de renforcement d'un concept d' «Indien » qui perdurera, en grande mesure, jusqu'à nos jours. Concrètement, trois processus ayant lieu au cours de ce débat ont été éclairés. Premièrement, bien que le terme «race » soit lentement tombé en désuétude au long de la décennie de 1940 comme un indicateur de l'altérité indienne, l'idée qu'il existe des traits héréditaires n'a pas tout à fait disparu ; cet héritage est maintenant désigné par le terme «culture », donnant ainsi lieu à une catégorie hybride qui n'arrive pas à se détacher du déterminisme du langage racial. Deuxièmement, l'idée suggérée par R. Redfield et J. de la Fuente selon laquelle au Mexique il n'y aurait pas de distinction nette (raciale ou autre) entre Indiens et non-Indiens restera marginale, à la faveur d'une «pensée différencialiste» qui établira l'altérité de ces groupes comme une prémisse. Cette idée réapparaîtra, néanmoins, non pas pour penser l'Indien mais pour penser le «métis» comme étant le sujet national par excellence dans ce qui plus tard s'appellera «idéologie du métissage », conception qui, théoriquement, garderait la société mexicaine du racisme caractéristique de son voisin du nord. Enfin, l'identification comme «Indien », deviendra, à la fin de ce débat, dépendante d'une appartenance à la collectivité, rendant pratiquement impossible de le concevoir comme un individu à part entière, indépendant de «sa » communauté27. L'altérisation repose, dès lors, non plus sur l'association avec un groupe racial ou ethnolinguistique, mais avec la «communauté », condition sine qua non pour le reconnaître comme tel. Idée toujours d'actualité, qui constitue, probablement, l'un des legs les plus durables de la politique indigéniste.

27. Résultat pour le moins paradoxal étant donné que ce sera précisément à cette époque que la migration vers les villes et vers les régions agro-industrielles commencera à s'élever significativement. 


\section{Version originale des citations}

[Épigraphe] Definir es, por esencia lógica, precisar las notas que convienen a un concepto no sólo para un momento dado, sino para siempre. Y precisamente la principal utilidad de la definición estriba en su permanencia.

[1] El número de individuos de la población continental que puedan ser correctamente clasificados con el término genérico de indios.

Deja al margen [...] a varios millones de individuos que solo hablan español pero son indígenas por su características étnicas y culturales.

Se necesita mucho tiempo, grandes elementos económicos.

Desde el punto de vista de su respectiva cultura, es decir, de acuerdo con los aspectos que presenta su vida material e intelectual.

[2] Una clasificación de características culturales que distinga entre características propiamente indígenas, [...] características extranjeras [...] y características mixtas.

Muy deficiente desarrollo biológico [correspondientes] a anacrónicas e inferiores etapas evolutivas.

[3] ¿Quién determina cuántas y cuáles [características] deben ser conservadas, extirpadas, corregidas o substituidas, y cuántas y cuáles introducidas? ¿Es conveniente dejar tan difícil tarea al exclusivo criterio del mismo indígena [...]?

[4] In such a country as Mexico, where much racial intermixture has occurred [...] one cannot enumerate the Indians in a biological sense. Nor it is possible to count the Indians as a socially recognized group, as we do with Negroes in the United States, because in most parts of Mexico Indians are not so defined [...] nobody is very definitely an Indian.

[5] The "Indian problem" of Mexico is the problem of converting many little folk societies into a nation.

[6] Han permanecido en un aislamiento tal que les ha sido posible conservarse como grupos étnicos diferenciados. Otras razas indígenas se han mezclado entre sí, pero el resultado [...] es siempre un tipo indio...

[7] Identificar en México, raza indígena y clase social, solo lleva a tergiversaciones interesadas [...] porque elimina, artificialmente, uno de sus términos principales: el de raza que juega en él un papel preponderante.

[8] In the greater part of Guatemala, present-day distinctions are not founded on biological race.

[9] There is an Indian problem in Guatemala, but to understand it, and solve it, one must first cleanse his mind of the idea that it is a race problem. It is true that by and large the people who are called Indians in Guatemala are blood- 
descendants of American aborigines; it is also true that most of the non-Indians in Guatemala have more or less European blood. It is not true that these facts are socially important.

[10] All of the criteria mentioned are independent of physical heritage [...] the whole question of blood... is irrelevant in the distinction actually made.

Once [this] is clearly understood [...] it need not seem strange that one of two brothers can be an Indian and the other, perhaps living in the same town, a Ladino.

Passage from one group to the other is possible; more than that, it tends to become automatic. An Indian who loses his Indian characteristics is a Ladino.

[11] The article seeks to abolish the factor of Indianhood from consideration, in treating of a country whose population is largely Indian [...] Indeed, it used to be customary to abolish the Indian problem in just this way.

[12] To insist that the Indian does not exist or, as an Indian, should be ignored, unless his biological, linguistic, social, legal and economic identification are exactly coterminal, would be to confuse the subject hopelessly.

[13] The total experience of countries like Mexico and the US...

[14] The important differences between groups in Guatemala are differences of culture. They are not differences of race [...] In the United States there is a social barrier between racial groups that is based on awareness of differences in blood between members of one race and members of another. This barrier is very strong in Negro-White relations; it is present in Indian-White relations. It is practically absent in much of Guatemala.

[15] Seems to presume that no inner or organic dynamic values exist within the Indian culture or life.

[16] If, then, by Indianhood [the administrators] mean not pre-Columbian customs and values but something that develops within native traditions and does not remain static or even stable, I see no great conflict with the anthropologists. For the latter does not maintain that acculturative forces will reduce the Indian to a dead level of national homogeneity.

[17] A definition that will be practical from the point of view of gathering statistical and cadastral data and usable in relation to the goals and purposes of the III. The determining characteristics of what is indigenous.

"Most acute economic and social deficiencies" of the social groups.

[18] According to our criterion, when a group has the maximum number of quantitative needs and deficiencies [...] alongside the maximum number of qualitative deficiencies, we are in the presence of a group that we might call indigenous. 
[19] Que sea necesario [...] definir con precisión, primeramente, los grupos que deben considerarse indios.

No siempre es conveniente dirigirse hacia el indio como tal [debido a que muchos de ellos] han pasado a ser no-indios.

[20] El "indio" no se ha considerado a sí mismo como tal o ha aprendido ya a considerarse un campesino, un trabajador, o un nativo de una región específica. Lo que tendería a permanecer sería la Indianidad derivada de una localidad o una región [ser de un pueblo o ser nieto de los de un pueblo].

[21] Hacen de [los indígenas] sujetos especiales, diferentes de la población “campesina” y más diferentes aun de la urbana.

[22] ¿Es lógico y posible que por el solo hecho de que los dos grupos de individuos [indígenas y no-indígenas] [...] se debaten en iguales o análogas condiciones de miseria, sea eficaz aplicarles el mismo método y tratamiento? [...] Es obvio que tal cosa resulta imposible, que hay que formular distintos tratamientos para ambos grupos y que el problema del Indígena existe y siempre ha existido.

[23] En un mismo pueblo, en una misma familia, uno de los hijos puede presentar caracteres somáticos indígenas que faltan en el otro y en cambio, éste tener otros caracteres indígenas que faltan en el primero.

[24] La conciencia de pertenecer o no a un grupo indígena es [...] el rasgo más importante [...] pero [...] el más difícil de captar.

[25] Es indio el que se siente pertenecer a una comunidad indígena. [...] Llegamos en consecuencia a considerar que [...] lo que importa determinar en una política indigenista [...], es, fundamentalmente, la comunidad indígena [que debe entenderse como] aquella en que predominan elementos somáticos no europeos [...] y que tiene un sentido social de comunidad aislada.

[26] Los grandes problemas del indio, por lo menos en México, no son sólo económicos, sino fundamentalmente culturales.

En suma, lo que falta que llevemos al indio para resolver sus problemas es cultura. 


\section{Sources primaires imprimées}

Anonyme, 1947, «Editorial», América indígena, vol.7, nº 4, p. 279-284.

CASo Alfonso, 1948, «Definición del indio y de lo indio », América Indígena, vol. 8, nº 4, p. 239-247.

Fuente Julio de la, 1947a, «Definición, pase y desaparición del indio en México », América Indígena, vol. $7, \mathrm{n}^{\circ} 1$, p. 63-69.

-, 1947b, «Discriminación y negación del indio », América Indígena, vol. 7, n³ 3, p. 211215.

Gamı Manuel, 1942a, «Consideraciones sobre el problema indígena en América », América Indígena, vol. 2, n² 2, p. 17-23.

-, $1942 \mathrm{~b}$, "Las características culturales y los censos indígenas », América Indígena, vol. 2, n' ${ }^{\circ}$, p. $15-19$.

-, 1942C, «Calificación de características culturales de los grupos indígenas », América Indígena, vol. 2, n 4 , p. 17-22.

LEWIS Óscar et MAES Ernest E., 1945, «Bases para una nueva definición práctica del indio », América Indígena, vol. 5, $\mathrm{n}^{\circ} 2$ 2, p. 107-118.

Mendieta y Núñez Lucio, 1942, «Notas sobre el artículo “El indio en México" de Robert Redfield », Revista Mexicana de Sociología, vol.4, n³ 3, p.63-68.

REDFIELD Robert, 1942, "Del pensamiento sociológico actual : el indio en México», Revista Mexicana de Sociología, vol.4, n³, p. 103-120.

Redfield, Collier, Sady et Presnall, 1943 «Comments on Dr. Tax’s Article», América Indigena, vol. 3, $\mathrm{n}^{\circ} 1, \mathrm{p} .83-90$.

STEWARD Julian, 1943, «Acculturation and the Indian Problem », América Indígena, vol. 3 , $n^{\circ} 4$, p. 323-328.

TAx Sol, 1942, «Ethnic relations in Guatemala », América Indígena, vol. 2, n 4, p. 42-48.

\section{Références}

AlvizURI Verushka, 2012, Le Savant, le militant et l'aymara. Histoire d'une construction identitaire (1952-2006), Paris, A. Colin.

CANESSA Andrew, 2012, Intimate Indigeneities, Durham, Duke University Press.

FAVRE Henri, 1996, L'Indigénisme, Paris, PUF.

FRADIN Bernard, QUÉRÉ Louis et WIDMER Jean éd., 1994, L'Enquête sur les catégories. De Durkheim à Sacks, Paris, Éd. de l’EHESS.

GIRAUDO Laura, 2014, "Entre "atraso estadístico" e "indigenismo científico" : Uniformar los censos y definir a los indígenas en las Américas ", La novedad estadística. Cuantificar, cualificar y transformar las poblaciones en Europa y América Latina, siglos XIXY XX, J. Bustamante, L. Giraudo et L. Mayer éd., Madrid, Ediciones Polifemo, p.127-197.

GIRAUdo Laura et MARTín-SÁnCHEZ Juan, 2013, «Dos debates medulares sobre el concepto de raza, 1943-1952 », Revista Mexicana de Sociología, vol.75, nº 4, p. 527-555.

GIUDICELLI Christophe, 2010, «Historia de un equívoco. La traducción etnográfica de las clasificaciones coloniales. El caso neovizcaíno », Fronteras Movedizas. Clasificaciones coloniales y dinámicas socioculturales en las fronteras americanas, C. Giudicelli éd., Mexico, Zamora, Colmich, CEMCA, p.139-171. 
GuYon Stéphanie, 2013, «Des "Primitifs” aux “Autochtones”. Savoirs ethnographiques et politiques publiques en Guyane de 1946 à nos jours », Genèses, nº 91, p. 49-70.

HewitT Cynthia, 1988, Imágenes del campo : interpretaciones antropológicas del México rural, Mexico, El Colegio de México.

KNIGHT Alan, 1990, «Racism, revolution and indigenismo. Mexico 1910-1940 », The Idea of Race in Latin America, R. Graham éd., Austin, University of Texas Press.

LEPETIT Bernard, 2013, Les Formes de l'expérience. Une autre histoire sociale, Paris, A. Michel.

LóPEZ CABALlero Paula, 2012, «Altérités intimes, altérités éloignées. La greffe du multiculturalisme au Mexique et en Amérique latine», Critique internationale, n51, p.129-149.

López Caballero Paula et Giudicelli Christophe, 2016, Régimes nationaux d'altérité. États-nations et altérités autochtones en Amérique latine, Rennes, PUR.

NUDLER Óscar, 2004, «Hacia un modelo de cambio conceptual : espacios controversiales y relocalización », Revista de Filosofía, vol. 29, n² 2, p.7-19.

TÉLLEz Ortega, 1987, «La época de oro (1940-1964) », La antropología en México, panorama histórico, vol. 2 Los hechos y los dichos (1880-1986), C. García Mora éd., Mexico, INAH, p. 291-338.

WADE Peter, 1993, " "Race”, Nature and Culture », Man, New Series, vol. 28, n¹, p. 17-34. 